\title{
EXISTENCE CONDITIONS FOR THE LANE INTEGRAL
}

\author{
KEITH P. SMITH AND FRED M. WRIGHT
}

1. Introduction. We deal in this paper with an integral which was defined by R. E. Lane [4] and which is an extension of the Stieltjes mean sigma integral introduced by H. L. Smith [6]. If $f$ and $g$ are real-valued functions on an interval $[a, b]$ of the real axis, and if $D=\left\{a=x_{0}<x_{1}<x_{2}<\cdots<x_{n}=b\right\}$ is a subdivision of $[a, b]$, we use the symbol $S_{D}(f, g)$ to denote the sum

$$
\sum_{i=1}^{n} \frac{f\left(x_{i-1}\right)+f\left(x_{i}\right)}{2} \cdot\left[g\left(x_{i}\right)-g\left(x_{i-1}\right)\right] .
$$

If $[a, b]$ is an interval of the real axis, and if $G$ is a subset of $[a, b]$ containing $a$ and $b$, we use the terminology $G$-subdivision of $[a, b]$ and $G$-refinement of a subdivision of $[a, b]$ as in [1]. The concepts of singular graph, exceptional number, and summability set are as in [4]. If $f$ and $g$ are real-valued functions on an interval $[a, b]$ of the real axis, and if there is a summability set $G$ for $f$ and $g$ in $[a, b]$, the Lane integral $L \int_{a}^{b} f d g$ is the refinement limit

$$
\lim _{D \subset G, \supset} S_{D}(f, g),
$$

and this integral is the Stieltjes mean sigma integral $F m \int_{a}^{b} f d g$ in case the entire interval $[a, b]$ is a summability set for $f$ and $g$ in $[a, b]$.

In $\S 2$ we deal with a bounded real-valued function $f$ on an interval $[a, b]$ of the real axis, a nondecreasing real-valued function $g$ on the entire real axis and a subset $G$ of $[a, b]$ dense on this interval and containing its endpoints. We suppose that if $H=(a, b)-G$ and $H$ is nonempty then the ordered triple $(g, f, H)$ is a singular graph, and that if $y$ is in $G$ and the restriction of $g$ to $[a, b]$ is discontinuous at $y$, then $y$ is not an exceptional number for $f$ and $g$ in $[a, b]$. We establish Theorem 2.1, which is analogous to Theorem 18 on page 278 of Graves [2] for the Stieltjes norm in tegral and the Stieltjes sigma integral, and which provides a set of necessary and sufficient conditions for $G$ to be a summability set for $f$ and $g$ in $[a, b]$. Theorem 2.1 clearly provides as a special case a set of necessary and sufficient conditions for the Stieltjes mean sigma integral $F m \int_{a}^{b} f d g$ to exist. We show how we have fairly simply from Theorem 2.1 the result due to Bzoch [1] that $G$ is a summability set for $f$ and $g$ in $[a, b]$ if and only if there is a bounded

Presented to the Society, August 30, 1966; received by the editors October 7, 1966. 
real-valued function $u$ on $[a, b]$ such that $u(x)=f(x)$ for all $x$ in $G$ and such that the Stieltjes mean sigma integral $F m \int_{a}^{b} u d g$ exists.

In $\S 3$ with $f, g, G$, and $H$ as in $\$ 2$ we use our Theorem 2.1 to obtain a necessary and sufficient condition for $G$ to be a summability set for $f$ and $g$ in $[a, b]$ which is analogous to one given by C. B. Murray [5] for the Stieltjes mean sigma integral.

2. An existence theorem. Suppose in this section that $f$ is a bounded real-valued function on an interval $[a, b]$ of the real axis, $g$ is a nondecreasing real-valued function on the entire real axis, $G$ is a subset of $[a, b]$ containing $a$ and $b$ which is dense on $[a, b]$, if $H=(a, b)-G$ and $H$ is nonempty then $(g, f, H)$ is a singular graph, and for each $y$ in $G$ if the restriction of $g$ to $[a, b]$ is discontinuous at $y$ then $y$ is not an exceptional number for $f$ and $g$ in $[a, b]$. If $a \leqq y<b$ let $f_{G}\left(y^{+}\right)$ denote the limit

$$
\lim _{x \rightarrow y^{+}, x \in G} f(x),
$$

and similarly define $f_{G}\left(y^{-}\right)$for $a<y \leqq b$. Let $M_{G}\left(f^{+}\right)$denote the set of all numbers $y$ such that $a \leqq y<b$ and $f_{G}\left(y^{+}\right)$does not exist, and similarly define $M_{G}\left(f^{-}\right)$. Then let

$$
M_{G}(f)=M_{G}\left(f^{+}\right)+M_{G}\left(f^{-}\right) .
$$

Also, let $s$ and $\psi$ be a nondecreasing saltus function on the entire real axis and a continuous nondecreasing function on the entire real axis, respectively, such that $g=s+\psi$. Let $\mu_{\psi}{ }^{*}$ denote the outer measure function associated with the nondecreasing function $\psi$.

TheOREM 2.1. For $G$ to be a summability set for $f$ and $g$ in $[a, b]$, it is necessary and sufficient that the following statements hold:

(a) if $a \leqq y<b$ and $g\left(y^{+}\right) \neq g(y)$, then $f_{G}\left(y^{+}\right)$exists;

(b) if $a<y \leqq b$ and $g\left(y^{-}\right) \neq g(y)$, then $f_{G}\left(y^{-}\right)$exists;

(c) $\mu_{\psi}{ }^{*}\left(M_{G}(f)\right)=0$.

Proof of THE SUfFiciency. We establish the sufficiency of the conjunction of (a), (b) and (c) by demonstrating that if these statements hold then

$$
\lim _{D \subset G, \supset} S_{D}(f, s) \text { and } \lim _{D \subset G, \supset} S_{D}(f, \psi)
$$

both exist, so that we can then conclude that

$$
\lim _{D \subset G, \supset} S_{D}(f, g)
$$

exists. 
Proceeding in a manner analogous to that used by Lane in his proof of Theorem 3.1 of [3], we can show that the conjunction of (a) and (b) implies that the limit

$$
\lim _{D \subset \tilde{G}, \supset} S_{D}(f, s)
$$

exists, where $\tilde{G}$ is the union of $G$ and the set of points of $(a, b)$ at which $g$ is discontinuous. Then using the continuity of $\psi$ in a modification of the sufficiency proof of Theorem 1 of [1] we can obtain the result that the conjunction of (a) and (b) implies that the limit

$$
\lim _{D \subset G, \supset} S_{D}(f, s)
$$

exists.

In this part of our sufficiency proof, we show that (c) implies that

$$
\lim _{D \subset G, \supset} S_{D}(f, \psi)
$$

exists. If $A$ is a nonempty subset of $G$, let

$$
\omega_{G}(A)=\underset{x \text { in } A}{\text { l.u.b. }} f(x)-\underset{x \text { in } A}{\operatorname{g.l.b}} f(x) .
$$

If $y$ is in $[a, b]$, let

$$
\omega_{G}(y)=\lim _{\delta \rightarrow 0^{+}} \omega_{G}((y-\delta, y+\delta) \cdot G) .
$$

For $\eta>0$, let $R_{G}(\eta)$ be the set of all numbers $y$ in $(a, b)$ such that $\omega_{G}(y) \geqq \eta, y \notin G$, and $y \notin M_{G}(f)$. If $\eta>0$, each point of $R_{G}(\eta)$ is an isolated point of this set, so that $R_{G}(\eta)$ is countable, and hence $\mu_{\psi}^{*}\left(R_{G}(\eta)\right)=0$. For $\eta>0$, let $T_{G}(\eta)$ denote the set of all $y$ in $[a, b]$ such that $\omega_{G}(y) \geqq \eta$. If $\eta>0, \mu_{\psi}^{*}\left(T_{G}(\eta)\right)=0$, and $T_{G}(\eta)$ is closed. Proceeding as in the proof of the analogous result for the Stieltjes integral we can show that the desired limit exists.

Proof of the neCEssity. By assumption $G$ is a summability set for $f$ and $g$ in $[a, b]$.

Suppose that (a) does not hold. Then there is a $y$ in $[a, b)$ such that $g\left(y^{+}\right) \neq g(y)$ and $f_{G}\left(y^{+}\right)$does not exist. Let $l=g\left(y^{+}\right)-g(y)$. There is a positive number $k$ for which if $y<z \leqq b$ then there are points $s$ and $t$ in $G \cdot(y, z)$ such that $|f(s)-f(t)| \geqq k$. Let $N$ be a positive number such that $|f(x)| \leqq N$ if $x \in[a, b]$. There is a number $w$ in $(y, b]$ such that if $x \in(y, w)$ then $\left|g(x)-g\left(y^{+}\right)\right|<\min \{l / 2, l k / 16 N\}$. Let $\bar{G}=G+\{y\}$. By Lemma 2 of $[1], \bar{G}$ is a summability set for $f$ and $g$ in $[a, b]$. Suppose that $P$ is a $\bar{G}$-subdivision of $[a, b]$. Let $\bar{P}$ be a $\bar{G}$-refinement of $P$ containing $y$ and at least one point of $(y, w)$. Let $z$ be the smallest 
number in $\bar{P} \cdot(y, w)$. Let $s$ and $t$ be in $G \cdot(y, z)$ such that $|f(s)-f(t)|$ $\geqq k$. Let $P^{\prime}=\bar{P}+\{s\}$ and $P^{\prime \prime}=\bar{P}+\{t\} . P^{\prime}$ and $P^{\prime \prime}$ are $\bar{G}$-refinements of $P$, and

$$
\begin{aligned}
\left|S_{P^{\prime}}(f, g)-S_{P^{\prime \prime}}(f, g)\right| & =\frac{1}{2}\{|f(s)-f(t)| \cdot|g(z)-g(y)| \\
& \quad-|f(z)-f(y)| \cdot|g(s)-g(t)|\} \\
& >l k / 8 .
\end{aligned}
$$

This contradicts the result that $\bar{G}$ is a summability set for $f$ and $g$ in $[a, b]$.

We have from the above that (a) holds. Similarly (b) holds.

We now argue indirectly to show that (c) holds. Since by hypothesis

$$
\lim _{D \subset G, \supset} S_{D}(f, g)
$$

exists and since it follows from the result that (a) and (b) hold that

$$
\lim _{D \subset G, \supset} S_{D}(f, s)
$$

exists, we can conclude that

$$
\lim _{D \subset G, \supset} S_{D}(f, \psi)
$$

exists. Suppose that $\mu_{\psi}^{*}\left(M_{G}(f)\right)>0$. Then at least one of $\mu_{\psi}^{*}\left(M_{G}\left(f^{+}\right)\right)$ and $\mu_{\psi}^{*}\left(M_{G}\left(f^{-}\right)\right)$is positive; suppose for the sake of argument that $\mu_{\psi}^{*}\left(M_{G}\left(f^{+}\right)\right)>0$. For each positive number $\delta$, let $M_{G}\left(f, \delta^{+}\right)$be the set of all $x$ in $[a, b)$ such that

$$
\left[\limsup _{t \rightarrow x^{+}, t \text { in } G} f(t)-\liminf _{t \rightarrow x^{+}, t \text { in } G} f(t)\right] \geqq \delta .
$$

There is a positive integer $\bar{\jmath}$ such that $\omega_{\bar{j}}^{+} \equiv \mu_{\psi}^{*}\left(M_{G}\left(f,(1 / \bar{\jmath})^{+}\right)\right)>0$. Let $P=\left\{a=x_{0}<x_{1}<\cdots<x_{n}=b\right\}$ be a $G$-subdivision of $[a, b]$. Let $\left\{i_{1}<i_{2}<\cdots<i_{m}\right\}$ be the set of all positive integers $i$ not exceeding $n$ such that the segment $\left(x_{i-1}, x_{i}\right)$ contains a point of $M_{G}\left(f,(1 / \bar{\jmath})^{+}\right)$. For $q=1,2, \cdots, m$, let $u_{q}=$ l.u.b. $M_{G}\left(f,(1 / \bar{j})^{+}\right) \cdot\left(x_{i_{q}-1}, x_{i_{q}}\right)$. Using the continuity of $\psi$ we can show that

$$
\sum_{q=1}^{m}\left[\psi\left(u_{q}\right)-\psi\left(x_{i q-1}\right)\right]>\frac{1}{2} \omega j^{+}
$$

For each $q=1,2, \cdots, m$ for which $u_{q}=x_{i_{q}}$, or else $u_{q}<x_{i_{q}}$ and $u_{q} \notin M_{G}\left(f,(1 / \bar{j})^{+}\right)$, let $v_{q}$ be a number in $\left(x_{i_{q}-1}, u_{q}\right)$ such that the inequality

$$
\left|\psi\left(u_{q}\right)-\psi(x)\right|<(1 / 4 m) \cdot \omega_{j}^{+}
$$


holds if $x \in\left(v_{q}, u_{q}\right)$, and such that the inequality

$$
\left|\psi\left(x^{\prime \prime}\right)-\psi\left(x^{\prime}\right)\right|<(1 / 32 m N) \cdot \omega_{\bar{j}}^{+} \cdot 1 / \bar{\jmath}
$$

holds if $x^{\prime}$ and $x^{\prime \prime}$ are in $\left(v_{q}, u_{q}\right)$. For each $q=1,2, \cdots, m$ for which $u_{q}<x_{i_{q}}$ and $u_{q} \in M_{G}\left(f,(1 / \bar{\jmath})^{+}\right)$, let $v_{q}$ be a number in $\left(u_{q}, x_{i_{q}}\right)$ such that (1) holds if $x \in\left(u_{q}, v_{q}\right)$, and such that (2) holds if $x^{\prime}$ and $x^{\prime \prime}$ are in $\left(u_{q}, v_{q}\right)$. For $q=1,2, \cdots, m$, let $s_{q}$ and $t_{q}$ be numbers in $G$ satisfying $v_{q}<s_{q}<t_{q}<u_{q}$ or $u_{q}<s_{q}<t_{q}<v_{q}$ according as $v_{q}<u_{q}$ or $u_{q}<v_{q}$, and such that $\left|f\left(t_{q}\right)-f\left(s_{q}\right)\right|>1 / 2 j$. For $q=1,2, \cdots, m$, let

$$
\begin{aligned}
d_{q}= & {\left[f\left(x_{i_{q}-1}\right)+f\left(t_{q}\right)\right]\left[\psi\left(t_{q}\right)-\psi\left(x_{i_{q}-1}\right)\right] } \\
& -\left\{\left[f\left(x_{i_{q}-1}\right)+f\left(s_{q}\right)\right]\left[\psi\left(s_{q}\right)-\psi\left(x_{i_{q}-1}\right)\right]\right. \\
& \left.+\left[f\left(s_{q}\right)+f\left(t_{q}\right)\right]\left[\psi\left(t_{q}\right)-\psi\left(s_{q}\right)\right]\right\} .
\end{aligned}
$$

Let $P_{1}$ be the $G$-refinement of $P$ such that $P_{1}=P+Q_{1}+\cdots+Q_{m}$ where for $q=1,2, \cdots, m, Q_{q}$ is $\left\{s_{q}, t_{q}\right\}$ or $\left\{t_{q}\right\}$ according as $d_{q}$ is nonnegative or negative, and let $P_{2}$ be the $G$-refinement of $P$ such that $P_{2}=P+R_{1}+\cdots+R_{m}$ where for $q=1,2, \cdots, m, R_{q}$ is $\left\{t_{q}\right\}$ or $\left\{s_{q}, t_{q}\right\}$ according as $d_{q}$ is nonnegative or negative. We have that

$$
\begin{aligned}
& \left|S_{P_{1}}(f, \psi)-S_{P_{2}}(f, \psi)\right|=\frac{1}{2} \sum_{q=1}^{m}\left|d_{q}\right| \\
& \geqq \frac{1}{2} \sum_{q=1}^{m}\left\{\left|f\left(t_{q}\right)-f\left(s_{q}\right)\right|\left[\psi\left(s_{q}\right)-\psi\left(x_{i_{q}-1}\right)\right]\right. \\
& \left.\quad \quad-\left|f\left(s_{q}\right)-f\left(x_{i_{q}-1}\right)\right|\left[\psi\left(t_{q}\right)-\psi\left(s_{q}\right)\right]\right\} \\
& >\frac{1}{2}\left\{(1 / 2 \bar{\jmath}) \cdot \frac{1}{4} \omega_{\bar{j}}^{+}-(2 N m / 32 m N) \cdot \omega^{\dagger} \cdot 1 / \bar{j}\right\} \\
& =(1 / 32) \omega_{j}^{+} \cdot 1 / \bar{\jmath} .
\end{aligned}
$$

This contradicts the result that $\lim _{D \subset G}, S_{D}(f, \psi)$ exists. We conclude that $\mu_{\psi}^{*}\left(M_{G}(f)\right)=0$.

We now show how the following result due to Bzoch [1] follows fairly simply from Theorem 2.1 .

TheOREM 2.2. For $G$ to be a summability set for $f$ and $g$ in $[a, b]$, it is necessary and sufficient that there exists a bounded real-valued function $u$ on $[a, b]$ such that $u(x)=f(x)$ for all $x$ in $G$ and such that the Stieltjes mean sigma integral Fm $\int_{a}^{b} u d g$ exists.

Proof of The Necessity. It is easy to determine a bounded realvalued function $u$ on $[a, b]$ such that

(i) $u(y)=f(y)$ if $y \in G$; 
(ii) if $a \leqq y<b$ and $f_{G}\left(y^{+}\right)$exists, then $u\left(y^{+}\right)$exists;

(iii) if $a<y \leqq b$ and $f_{G}\left(y^{-}\right)$exists, then $u\left(y^{-}\right)$exists.

Let $D$ be the set of points of discontinuity of $u$. Since $\mu_{\psi}^{*}\left(M_{G}(f)\right)=0$ in view of Theorem 2.1 , it follows that $\mu_{\psi}^{*}(D)=0$. It follows, then, from Theorem 2.1 that $F m \int_{a}^{b} u d g$ exists.

ProOF of THE SUfFICIENCY. By assumption there is a bounded real-valued function $u$ on $[a, b]$ such that $u(x)=f(x)$ for all $x$ in $G$, and such that the Stieltjes mean sigma integral $F m \int_{a}^{b} u d g$ exists. Let $D$ be the set of points of discontinuity of $u$. From Theorem 2.1 we have that $\mu_{\psi}^{*}(D)=0 . M_{G}(f) \subset D$; hence $\mu_{\psi}^{*}\left(M_{G}(f)\right)=0$. It follows, then, from Theorem 2.1 that the Lane integral $L \int_{a}^{b} f d g$ exists.

3. A further existence theorem. Let the functions $f, g, s, \psi, \omega_{G}$ and the sets $G, H$ be as in $\S 2$. In the next theorem we present a necessary condition for $G$ to be a summability set for $f$ and $g$ in $[a, b]$ which is analogous to one given by C. B. Murray [5] for the Stieltjes mean sigma integral. Using the hypothesis that $(g, f, H)$ is a singular graph in a modification of the sufficiency proof of Theorem 1.2 of [5] we can show that the condition of the following theorem is also sufficient.

THEOREM 3.1. Suppose that $G$ is a summability set for $f$ and $g$ in $[a, b]$. Then for positive numbers $\epsilon$ and $\eta$ there is a subdivision $D$ $=\left\{a=y_{0}<y_{1}<\cdots<y_{n}=b\right\}$ of $[a, b]$ with the property that if $\left\{i_{1}<i_{2}<\cdots<i_{p}\right\}$ is the set of all positive integers $i$ not exceeding $n$ for which there are points $x^{\prime}, x^{\prime \prime}$ in $\left(y_{i-1}, y_{i}\right) \cdot G$ such that $\left|f\left(x^{\prime}\right)-f\left(x^{\prime \prime}\right)\right|$ $\geqq \eta$, then $\sum_{j=1}^{p}\left[g\left(y_{i_{j}}\right)-g\left(y_{i_{j}-1}\right)\right]<\epsilon$.

Proof. We have from the sufficiency proof of Theorem 2.1 that there are a $G$-subdivision $\tilde{D}=\left\{a=t_{0}<t_{1}<\cdots<t_{m}=b\right\}$ of $[a, b]$ and a subset $\tilde{L}=\left\{k_{1}<k_{2}<\cdots<k_{q}\right\}$ of the set of all positive integers not exceeding $m$ such that

$$
\sum_{j=1}^{q}\left[\psi\left(t_{k_{j}}\right)-\psi\left(t_{k_{j-1}}\right)\right]<\epsilon / 2
$$

and such that if $k$ is a positive integer not exceeding $m$ such that $k$ is not in $\tilde{L}$ then $\omega_{G}\left(\left[t_{k-1}, t_{k}\right] \cdot G\right)<\eta$.

Let $j$ be a positive integer not exceeding $q$. Let $\left\{z_{i, j}\right\}_{i=1}^{\infty}$ be a sequence of distinct points of $\left[t_{k_{j}-1}, t_{k_{j}}\right]$ containing all of the points of this interval at which the saltus function $s$ is discontinuous. For simplicity, let $z_{1, j}=t_{k_{j}-1}$ and $z_{2, j}=t_{k_{j}}$. Let $\bar{\imath}$ be a positive integer such that

$$
\sum_{i=i+2}^{\infty}\left[s\left(z_{i, j}^{+}\right)-s\left(\overline{z_{i, j}}\right)\right]<\epsilon / 2 q .
$$


Let $\Delta_{j}=\left\{t_{k_{j-1}}=u_{0, j}<u_{1, j}<\cdots<u_{i, j}=t_{k_{j}}\right\}$ be the subdivision of $\left[t_{k_{j}-1}, t_{k_{j}}\right]$ consisting of the numbers $z_{1, j}, z_{2, j}, \cdots, z_{i+1, j}$. For $i=1,2$, $\cdots, \bar{i}$, let $u_{i, j}^{\prime}$ be a number satisfying $u_{i-1, j}<u_{i, j}^{\prime}<u_{i, j}$, and such that $\omega_{G}\left(\left(u_{i-1, j}, u_{i, j}^{\prime}\right) \cdot G\right)<\eta$ in case $g\left(u_{i-1, j}^{+}\right) \neq g\left(u_{i-1, j}\right)$, and let $u_{i, j}^{\prime \prime}$ be a number satisfying $u_{i, j}^{\prime}<u_{i, j}^{\prime \prime}<u_{i, j}$, and such that $\omega_{G}\left(\left(u_{i, j}^{\prime \prime}, u_{i, j}\right) \cdot G\right)<\eta$ in case $g\left(u_{i, j}^{-}\right) \neq g\left(u_{i, j}\right)$. Let $\widetilde{D}_{j}=\left\{t_{k_{j}-1}=t_{0, j}<t_{1, j}<\cdots<t_{p_{j}, j}=t_{k_{j}}\right\}$ be the subdivision of $\left[t_{k_{j-1}}, t_{k_{j}}\right]$ consisting of the points of $\Delta_{j}$, the numbers $u_{1, j}^{\prime}, u_{2, j}^{\prime}, \cdots, u_{i, j}^{\prime}$, and the numbers $u_{1, j}^{\prime \prime}, u_{2, j}^{\prime \prime}, \cdots, u_{i, j}^{\prime \prime}$. Let $\left\{i_{1, j}<i_{2, j}<\cdots<i_{r_{j}, j}\right\}$ be the set of all positive integers $i$ not exceeding $p_{j}$ with $\omega_{G}\left(\left(t_{i-1, j}, t_{i, j}\right) \cdot G\right) \geqq \eta$. We have that

$$
\begin{aligned}
\sum_{l=1}^{r_{j}}\left[s\left(t_{i_{l, j}, j}\right)-s\left(t_{i_{l, j}-1, j}\right)\right] & \leqq \sum_{i=1}^{i}\left[s\left(\overline{u_{i, j}}\right)-s\left(u_{i-1, j}^{+}\right)\right] \\
& =\sum_{i=1}^{i} \sum_{l: u_{i-1, j}<z_{l, j}<u_{i}, j}\left[s\left(z_{l, j}^{+}\right)-s\left(\overline{z_{l, j}}\right)\right] \\
& =\sum_{i=i+2}^{\infty}\left[s\left(z_{i, j}^{+}\right)-s\left(\overline{z_{i, j}}\right)\right]<\epsilon / 2 q,
\end{aligned}
$$

Let $D=\left\{a=y_{0}<y_{1}<\cdots<y_{n}=b\right\}$ be the subdivision of $[a, b]$ equal to $\tilde{D}+\sum_{j=1}^{q} \widetilde{D}_{j}$. Let $\left\{j_{1}<j_{2}<\cdots<j_{r}\right\}$ be the set of all positive integers $j$ not exceeding $n$ such that $\omega_{G}\left(\left(y_{j-1}, y_{j}\right) \cdot G\right) \geqq \eta$. Then it follows that

$$
\begin{aligned}
\sum_{i=1}^{r}\left[g\left(y_{j_{i}}\right)-g\left(y_{j_{i}-1}\right)\right] \leqq \sum_{j=1}^{q} & {\left[\psi\left(t_{k_{j}}\right)-\psi\left(t_{k_{j}-1}\right)\right] } \\
& +\sum_{j=1}^{q} \sum_{l=1}^{r_{j}}\left[s\left(t_{i_{l, j}, j}\right)-s\left(t_{i_{l, j}-1, j}\right)\right]<\epsilon .
\end{aligned}
$$

REFERENCES

1. R. C. Bzoch, Existence conditions for an integral of R. E. Lane, J. Indian Math. Soc. 23 (1959), 117-124.

2. L. M. Graves, The theory of functions of real variables McGraw-Hill, New York, 1946.

3. R. E. Lane, The integral of a function with respect to a function, Proc. Amer. Math. Soc. 5 (1954), 59-66.

4. - The integral of a function with respect to a function. II, Proc. Amer. Math. Soc. 6(1955), 392-401.

5. C. B. Murray, On the mean integral, Ph.D. thesis, Univ. of Texas Library, Austin, Texas, 1964.

6. H. L. Smith, On the existence of the Stieltjes integral, Trans. Amer. Math. Soc. 27 (1925), 491-515.

OMAHA UNIVERSITY AND Iowa State University 